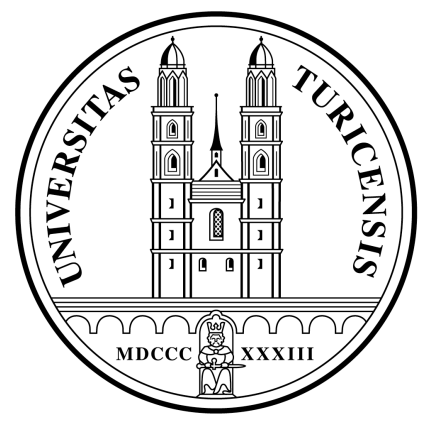

Institute for Empirical Research in Economics

University of Zurich

Working Paper Series

ISSN 1424-0459

Working Paper No. 66

Financial Markets and Stochastic Growth

Leonard J. Mirman and Klaus Reiner Schenk-Hoppé

November 2000 


\title{
Financial Markets and Stochastic Growth
}

\author{
Leonard J. Mirman \\ Department of Economics \\ University of Virginia, U.S.A. \\ Klaus Reiner Schenk-Hoppé \\ Institute for Empirical Research in Economics \\ University of Zurich, Switzerland
}

November 21, 2000

\begin{abstract}
In this paper, we study the effect of financial markets on the investment of a two-good two-country economy with stochastic production in a dynamic framework. Each country produces and invests only one good and, therefore, makes decisions as a central planner in an optimal growth model. Trade between consumers of both countries, however, takes place on competitive (spot or financial) markets. We compare the investment-consumption decisions of both 'market' models with the benchmark-case of an integrated world-equilibrium. In the log-linear case, we can uniquely characterize the state-dependent preferences of consumers that lead to dynamically efficient investment decisions. We show that the investment decisions in both 'market' models are, in general, inefficient as compared with the efficient, or integrated world economy, case.
\end{abstract}

\section{Introduction}

It is a well-known fact of economic theory that an Arrow-Debreu equilibrium is simply a reinterpretation of the Walrasian equilibrium concept to contingent commodities, see Arrow [1], Debreu [5], Radner [8]. Using these 
ideas one can study the allocation of risk in a general equilibrium framework. Indeed in this context, complete financial markets with contingent claims lead to Pareto-optimal allocations. Although there has been much literature on the effect of various market structures on asset allocation and their welfare effects in an uncertain environment, the question of capital accumulation in a dynamic general equilibrium model under uncertainty has not been studied. For example, it is not known whether the existence of complete financial markets, which generate Pareto-optimal allocations in the Arrow-Debreu framework, generate efficient dynamic solutions.

Although an analysis of this question in a competitive framework remains open due to the difficulties of studying infinite horizon general equilibrium models, this is a natural question in the context of an optimal growth model. Moreover, since in this type of model there must be several goods in order to trade and several countries each deciding on their own amount of investment, a natural setting for this question is the type of mixed economy, studied by Datta and Mirman [4]. Indeed, in the present paper we study the effect of financial markets on the investment of the economy when there are two countries, two goods and two states of the world. In this context we show that the investment cannot, in general (in particular when there exist statedependent preferences), be efficient - even in the face of complete financial markets. The use of a model with each country having a mixed economy depends on two things. The first is that each country produces and invests (or equivalently decides on aggregate consumption of) their own good, i.e., makes decisions as a central planner in an optimal growth model and, the second is, for each good (or country) there is a representative agent trading both goods in a Walrasian context. These agents, one from each country, use the aggregate consumption choice from the central planner as the initial endowment in the trading environment. This use of the Walrasian paradigm is a natural setting in which to use the Arrow-Debreu contingent claims framework.

This type of mixed economy model was used by Datta and Mirman to study a trade economy. Their model is without uncertainty but contains production externalities. It was shown there that the inefficiency associated with production externalities studied in Fischer and Mirman [6] can be mitigated if it is possible to internalize the production externalities by trade, through the preferences of the representative agents. In our model inefficiencies are also generated but these are due to the uncertainty, not to production externalities, and the assumption that tastes are state-dependent. Methods of 
Fischer and Mirman [6] are used to study these problems and are used in this paper as well.

In this paper, as in Datta and Mirman [4], we consider the relationship between a integrated world economy -in which there is one central plannerand the allocations generated by various assumptions on the type of markets. In the case of these different market types, each country must have a central planner to decide on its own aggregate consumptions and thus a differential game is entailed. This is the dynamic investment game between the two central planners. Indeed we study two cases of markets. The first is complete financial markets in which risk is allocated efficiently. The second case is when there are only spot markets -so that in general risk is not allocated efficiently. In both cases, we find an indirect utility function which the planners use to determine the optimal aggregate consumption for the representative agent in their country. This optimal aggregate consumption is used in turn by the representative agent as initial endowments in trading with the representative agent of the other country. Each country's representative agent takes the price as given in the trading process. However, the central planner of each country does not take the price as given.

We restrict the analysis to a log-linear economy so that there is a (computable) solution to the differentiable game between the two central planners. We show that in the case of only spot markets allocations are only Paretooptimal for particular specifications of the parameters while in the complete financial market case all allocations derived from trade are Pareto-optimal. We compare the investment-consumption decisions of the three models and show that the complete financial market case and the spot market case lead to the same but inefficient investment choices, i.e., the investment choices are different from that in the integrated world economy. This difference occurs because in the two 'market' cases there appears a consumption externality. These consumption externalities are taken account of in the case of the cooperative solution. Therefore the market investment choices are inefficient solutions. In fact, these inefficiencies are only possible if the preferences are state dependent. More generally, only if the tastes of the consumers are related in a very special way -making them both essentially the same- or if the preferences are independent of the state of the world are the 'market' cases efficient. This is similar to the results of Datta and Mirman [4] where the markets are able to internalize the production externalities under conditions that imply similar tastes. However, in the dynamic model there are no markets to perform that function so that tastes must be 'almost' equivalent for 
the two countries. Finally we show how the long-run equilibrium is related in these different cases.

The underlying static one-period models are studied Section 2. We derive the allocation of consumption goods in the non-cooperative cases of only spot markets (Section 2.1) and complete financial market (Section 2.2) as well as in the cooperative case (Section 2.3). Efficiency of the allocations is discussed in Section 2.4. In Section 3, we analyze the corresponding dynamic cases in which the central planner in each country decides strategically on the aggregate output, Section 3.1-3.3. Dynamic efficiency and the long-run behavior of the equilibrium is studied in Section 3.4.

\section{The One-Period Model}

Consider a world economy with two countries and two goods in which consumers' endowments and tastes depend on an exogenously determined state of nature. Each country $i(i=1,2)$ is endowed with only one good $i$. The consumers of each country, however, derive utility from consuming both goods, in each state of nature, and thus there is an incentive to trade, on spot as well as on financial markets. We assume that the population of each country is large and, therefore, consumers trade on competitive markets, i.e. each consumer takes prices as given. The time structure of trade is as follows. Financial markets, in which assets are traded, open at the beginning of the period, before the resolution of uncertainty. Spot markets, in which consumption goods are exchanged, open after the state of the world is revealed, and consumption takes place at the end of the period. Trading possibilities of consumers depend on the particular markets that are available to them. For instance, without financial markets, consumers cannot insure themselves against endowment shocks and variations in taste because wealth cannot be transferred across states of nature. The allocations on both the financial and the spot markets are determined by a Walrasian equilibrium with a pricetaking representative agent in each country. This agent maximizes expected utility when trading on the financial market and state-dependent utility when trading on the spot market.

We consider two extreme cases. In the first case, spot markets open in each state of the world after the resolution of uncertainty and there are no financial markets in which consumers can insure their wealth. In the other case, a complete financial market opens before the state of the world 
is revealed and spot markets open after the resolution of uncertainty. In the latter case consumers can insure themselves by transferring wealth across the states of nature. We derive the allocation of consumption goods in the world economy at the end of the period for both cases. The allocations are compared with the Pareto-optimal benchmark-allocation that is chosen by a benevolent social planner to maximize weighted total utility of the world economy.

We impose specific assumptions on the fundamentals to keep the analysis simple and to obtain explicit solutions throughout the paper. Assume that two states of nature $s(s=1,2)$ can occur with respective probability $\pi_{s}>0$ $\left(\pi_{1}+\pi_{2}=1\right)$. The representative agent in country $i$ has state-dependent initial endowment $\left(x_{i}(1), x_{i}(2)\right)$ and state-dependent preferences, described by the utility function,

$$
u_{i}\left(s, c_{i i}, c_{i j}\right)=\nu_{i i}(s) \log c_{i i}+\nu_{i j}(s) \log c_{i j}, \quad \nu_{i i}(s)>0, \nu_{i j}(s)>0,
$$

where $j=1,2$ and $j \neq i . \quad c_{i j}$ is the amount of good $j$ consumed by the representative agent of country $i$ at the end of the period.

\subsection{Spot Market Case}

Here we derive the allocation of consumption goods among the representative agents of the two countries if there exists no financial market and consumers can only trade on competitive spot markets after the state of the world is observed. Assuming that consumers take prices as given, the utility-maximization problem of the representative agent in country $i$ is as follows. If state $s$ is revealed, the representative agent in country $i$ chooses a consumption bundle such that,

$$
\begin{gathered}
\max _{\left(c_{i i}(s) \geq 0, c_{i j}(s) \geq 0\right)} \nu_{i i}(s) \log c_{i i}(s)+\nu_{i j}(s) \log c_{i j}(s) \\
\text { s.t. } p_{i}(s) c_{i i}(s)+p_{j}(s) c_{i j}(s) \leq p_{i}(s) x_{i}(s),
\end{gathered}
$$

where $j=1,2$ and $j \neq i$. For each state $s, p_{i}(s)$ is the spot market price of good $i$, and $c_{i j}(s)$ is the amount of good $j$ consumed by the representative agent in country $i . x_{i}(s)$ is the aggregate consumption of good $i$ in state $s$ as well as the endowment of the representative agent in country $i$ when trading in the spot market. 
The solution to this utility maximization problem determines the demand for goods of consumers in both countries in both states. One finds that,

$$
c_{i j}(s)=\frac{\nu_{i j}(s)}{\nu_{i 1}(s)+\nu_{i 2}(s)} \frac{p_{i}(s)}{p_{j}(s)} x_{i}(s) .
$$

From the market-clearing condition,

$$
c_{1 i}(s)+c_{2 i}(s)=x_{i}(s), \quad \text { for } i=1,2,
$$

we obtain the relative price $p(s)=p_{2}(s) / p_{1}(s)$ that clears the market:

$$
p(s)=\frac{\nu_{12}(s)\left(\nu_{21}(s)+\nu_{22}(s)\right)}{\nu_{21}(s)\left(\nu_{11}(s)+\nu_{12}(s)\right)} \frac{x_{1}(s)}{x_{2}(s)} .
$$

The market-clearing relative price depends on the preferences of both consumers in state $s$ and on the ratio of the initial endowments in that state. The relative price for good 2 in state $s$ increases, if total endowment in good 2 decreases, the preference in country 2 (country 1 ) for the foreign good decreases (increases), or the preference in country 2 (country 1 ) for the domestic good increases (decreases).

Thus, the allocation of consumption goods after trade is,

$$
c_{i j}^{S}(s)=\frac{\nu_{j i}(s)}{\nu_{j 1}(s)+\nu_{j 2}(s)} x_{j}(s) .
$$

The amount of good $j$ actually consumed by the representative agent in country $i$ is determined by the preferences of country $j$ and the initial endowment of country $j$. The share of the aggregate consumption of good $j$ owned by country $i$ at the end of the period is given by the relative weight that country $j$ assigns to good $i$. Thus, from each country's perspective, the amount of foreign goods consumed is determined only by the foreign country's preferences and initial endowment while the amount of domestic goods consumed depends only on the own preferences and initial endowment.

\subsection{Financial Market Case}

Here, we analyze the allocation of goods when consumers have access to a competitive financial market with a complete asset structure. In this market, contracts are traded for the receipt and delivery of goods after the resolution 
of uncertainty. Therefore, consumers can transfer wealth across states before the state of the world is revealed. After the uncertainty is resolved, deliveries and receipts of goods take place according to the portfolio of assets that consumers hold and, afterwards, spot markets for both goods open. Given their wealth in the prevailing state of nature, consumers finally allocate goods for consumption by trading in the spot market. It is assumed that consumers perfectly anticipate the spot prices while trading on the financial market. It is a well-known result, $[1,8]$, that this allocation of goods is identical to the one derived when consumers trade on a complete market for contingent commodities at the beginning of the period and no spot markets open afterwards. This is an implication of the Pareto-optimality of allocations, which holds if financial markets are complete. A detailed discussion is given in Section 2.4. Due to analytical tractability, we resort to this Arrow-Debreu framework in the following. We assume that the representative agent has objective probabilities and maximizes expected utility when trading in the financial market. Therefore the agent chooses a bundle of contingent commodities as follows,

$$
\begin{gathered}
\max _{\left(c_{i i}(s) \geq 0, c_{i j}(s) \geq 0\right)_{s=1,2}} \sum_{s=1}^{2} \pi_{s}\left(\nu_{i i}(s) \log c_{i i}(s)+\nu_{i j}(s) \log c_{i j}(s)\right), \\
\text { s.t. } \sum_{s=1}^{2}\left(p_{i}(s) c_{i i}(s)+p_{j}(s) c_{i j}(s)\right) \leq \sum_{s=1}^{2} p_{i}(s) x_{i}(s) .
\end{gathered}
$$

Here $c_{i j}(s)$ is the amount of good $j$ the representative agent $i$ consumes in state $s$. The state-dependent aggregate consumption at the end of the period is $x_{i}(s), i=1,2$.

The solution to the expected utility maximization problem (6) is,

$$
c_{i j}(s)=\frac{\pi_{s} \nu_{i j}(s)}{\mathbb{E}\left(\nu_{i 1}+\nu_{i 2}\right)} \frac{w_{i}}{p_{j}(s)}=: a_{i j}(s) \frac{w_{i}}{p_{j}(s)},
$$

where $\mathbb{E} \nu_{i j}=\pi_{1} \nu_{i j}(1)+\pi_{2} \nu_{i j}(2)$, denotes the mean value of country $i$ 's preferences for good $j$, and $w_{i}=p_{i}(1) x_{i}(1)+p_{i}(2) x_{i}(2)$, is the wealth of country $i$ while trading in contingent commodities.

To calculate the market-clearing prices, we insert the demand for contingent commodities (7) into the condition of market clearance $c_{i i}(s)+c_{j i}(s)=$ $x_{i}(s)$, for $i, j, s=1,2$ and $j \neq i$. By (7), the market-clearing condition is equivalent to,

$$
a_{i i}(s) w_{i}+a_{j i}(s) w_{j}=x_{i}(s) p_{i}(s) .
$$


We take good 1 in state 1 as numeraire, normalizing prices by $p_{1}(1)=1$. It is straightforward to check that solving (8) for the market-clearing prices is equivalent to solving the affine system,

$$
\left(\begin{array}{ccc}
a_{11}(1) & a_{21}(1) & a_{21}(1) \\
a_{12}(1) & a_{22}(1)-1 & a_{22}(1) \\
a_{11}(2)-1 & a_{21}(2) & a_{21}(2)
\end{array}\right)\left(\begin{array}{c}
\frac{x_{1}(2)}{x_{1}(1)} p_{1}(2) \\
\frac{x_{2}(1)}{x_{1}(1)} p_{2}(1) \\
\frac{x_{2}(2)}{x_{1}(1)} p_{2}(2)
\end{array}\right)=\left(\begin{array}{c}
1-a_{11}(1) \\
-a_{12}(1) \\
-a_{11}(2)
\end{array}\right) .
$$

The solution to this system is,

$$
p_{i}(s)=d_{i s} \frac{x_{1}(1)}{x_{i}(s)} \quad i, s=1,2,
$$

where the coefficients $d_{i s}$ depend only on the constants $a_{i j}(s)$. One has,

$$
\begin{aligned}
d_{11} & =1 \\
d_{12} & =\frac{\pi_{2}}{\pi_{1}} \frac{\nu_{21}(2) \mathbb{E}\left(\nu_{11}+\nu_{12}\right)-\pi_{1}\left[\nu_{11}(1) \nu_{21}(2)-\nu_{11}(2) \nu_{21}(1)\right]}{\nu_{21}(1) \mathbb{E}\left(\nu_{11}+\nu_{12}\right)+\pi_{2}\left[\nu_{11}(1) \nu_{21}(2)-\nu_{11}(2) \nu_{21}(1)\right]} \\
d_{21} & =\frac{\nu_{22}(1) \mathbb{E} \nu_{12}+\nu_{12}(1) \mathbb{E} \nu_{21}}{\nu_{21}(1) \mathbb{E}\left(\nu_{11}+\nu_{12}\right)+\pi_{2}\left[\nu_{11}(1) \nu_{21}(2)-\nu_{11}(2) \nu_{21}(1)\right]} \\
d_{22} & =\frac{\pi_{2}}{\pi_{1}} \frac{\nu_{22}(2) \mathbb{E} \nu_{12}+\nu_{12}(2) \mathbb{E} \nu_{21}}{\nu_{21}(1) \mathbb{E}\left(\nu_{11}+\nu_{12}\right)+\pi_{2}\left[\nu_{11}(1) \nu_{21}(2)-\nu_{11}(2) \nu_{21}(1)\right]}
\end{aligned}
$$

The allocation of consumption goods in equilibrium is therefore given by,

$$
c_{i j}^{F}(s)=\frac{\pi_{s} \nu_{i j}(s)}{\mathbb{E}\left(\nu_{i 1}+\nu_{i 2}\right)} \frac{d_{i 1}+d_{i 2}}{d_{j s}} x_{j}(s) .
$$

In the financial market case, the amount of good $j$ actually consumed by the representative agent in country $i$ in state $s$ depends on the preferences of both countries as well as on the probability of the state and the initial endowment $x_{j}(s)$.

\subsection{Cooperative Case}

We derive the optimal cooperative solution next. In this case, a single benevolent planner chooses a feasible state-dependent allocation of consumption goods for both representative agents. The objective of the planner is to maximizes the weighted sum of the expected utilities of the agents in both 
countries. Neither financial nor spot markets open in the cooperative case. The allocation, which is Pareto-optimal, and the associated utility levels provides an important benchmark for the market cases. One can thus determine whether the allocation in the world economy is a social optimum. The problem of the planner is to choose an allocation after the state is revealed to maximize the sum of the weighted utility of both representative agents in that state.

Given aggregate consumption levels $\left(x_{1}(s), x_{2}(s)\right)_{s=1,2}$ in the two countries, the objective of the social planner is to choose a feasible allocation $c_{i j}(s), i, j, s=1,2$ such that,

$$
\begin{aligned}
& \max _{\left(c_{i j}(s) \geq 0\right)_{i, j, s=1,2}} \sum_{i, j, s=1}^{2} \pi_{s} \mu_{i} \nu_{i j}(s) \log c_{i j}(s), \\
& \text { s.t. } c_{i i}(s)+c_{j i}(s) \leq x_{i}(s), \text { for all } i, j, s=1,2, j \neq i .
\end{aligned}
$$

$\mu_{i}>0$ is the relative weight assigned to the representative agent of country $i$. Let $\mu=\mu_{1}$, and $\mu_{2}=1-\mu$.

The solution to this maximization problem is,

$$
c_{i j}^{C}(s)=\frac{\mu_{i} \nu_{i j}(s)}{\mu_{1} \nu_{1 j}(s)+\mu_{2} \nu_{2 j}(s)} x_{j}(s) .
$$

This allocation is Pareto-optimal for any weight. The share of aggregate consumption of good $j$ in country $i$ is determined by the weighted preference of the representative agent in country $i$ for good $j$ relative to the total weighted preference of both countries for that good. The country with the higher weighted preference for a good obtains the larger share.

\subsection{Efficiency of Allocations}

We can now study the efficiency of the outcome in the two market cases. We compare the competitive equilibrium in each of the market cases to the solution in the benchmark case. Since the allocations in all three cases are generically different, it is, in general, not of interest to compare the resulting allocations in these three cases directly. More important are the welfare aspects associated with the different market structures.

It is a well-known result that the allocation derived in the case of complete competitive financial markets is Pareto-optimal. At least one consumer is 
reluctant to agree to a different allocation of consumption goods (or initial endowments) in any such equilibrium: there is no redistribution of goods such that at least one consumer is better off and the other consumer is not worse off. The cooperative case obviously also generates a Pareto-optimal allocation. However, if consumers can only trade on spot markets after the state of the world is revealed, the allocation is, in general, not Pareto-optimal. Thus, there may be an incentive for both representative agents to agree to a redistribution of goods, or to favor the introduction of financial markets.

It is of interest to know whether the state-dependence of preferences or endowments causes the inefficiency of the spot-market outcome. Assume that the preferences are independent of the state. Then, as elementary calculations show, the allocations in the spot market and the financial market case are identical in this example, i.e. $c_{i j}^{S}(s)=c_{i j}^{F}(s)$ for all $i, j, s=1,2$. Therefore, the lack of Pareto-optimality of the spot market allocation can be only due to the state-dependence of preferences.

However, the outcome of the no-financial-market case is not generically inefficient as the following result shows.

Lemma 2.1 The allocation $\left(c_{i j}^{S}(s)\right)_{i, j, s=1,2}$ in the spot market case is Paretooptimal if and only if the preferences of the representative agents in both countries satisfy

$$
\frac{\nu_{12}(1)}{\nu_{12}(2)}=\frac{\nu_{21}(1)}{\nu_{21}(2)}
$$

Proof. It suffices to show that there exists a weight $\mu$ such that the allocation in the spot market case is identical to that in the cooperative case. Then Pareto-optimality of the spot market allocation follows readily. An elementary calculation shows that $c_{i j}^{S}(s)=c_{i j}^{C}(s)$ for all $i, j, s=1,2$ if and only if (12) is satisfied. The weight is given by $\mu /(1-\mu)=\nu_{21}(1) / \nu_{12}(1)=\nu_{21}(2) / \nu_{12}(2)$.

Lemma 2.1 states that even when consumers do not have access to a financial market, the allocation of goods derived only through trade in spot markets is efficient, provided that the relative preference for the foreign good in the states of nature is identical in both countries. This holds in particular if the preference for the foreign good does not depend on the state of nature in both countries. The relative taste for the domestic good, however, may vary with the state.

Pareto-optimality in the spot market case has important implications. If the prevailing allocation is Pareto-optimal, at least one representative agent 
is reluctant to trade in the financial market; he offers his good only in the spot market after the state is observed. According to Lemma 2.1, trade of both agents in the financial market would lead to a different Pareto-optimal allocation, leaving one consumer with a lower level of utility. One might object that this situation is not of importance, because condition (12) only holds on a subset of the parameter space of Lebesgue-measure zero, i.e. it is the generic case that (12) does not hold. However, Pareto-optimality of the spot-market allocation is not necessary to observe opposition of one agent to the creation of financial markets. One has the following technical result which shows that opening a financial market in addition to spot markets is not necessarily welfare-improving.

Lemma 2.2 There exists a set $N \subset \mathbb{R}_{++}^{8}$ of positive Lebesgue-measure such that for each $\left(\nu_{i j}(s)\right)_{i, j, s=1,2} \in N$ one agent prefers not to trade in the financial market.

Proof. We show that the creation of a financial market makes one agent worse off for all preferences $\left(\nu_{i j}(s)\right)_{i, j, s=1,2} \in N$, where $N$ is a set of positive Lebesgue-measure which contains an element that fulfills (12). The proof uses a continuity argument.

Fix preference parameters, $\bar{\nu}:=\left(\nu_{i j}(s)\right)_{i, j, s=1,2} \in \mathbb{R}_{++}^{8}$, such that (12) holds and that the allocations in the spot and financial market case are different and lead to different levels of utility. Let the representative agent in country 1 be better off in the financial market case than in the spot market case. Thus agent 2 is against the opening of the financial market.

For all parameters close to the initial parameter $\bar{\nu}$, the utility of each agent is close to his initial utility. This follows from continuity of allocations and utility in $\left(\nu_{i j}(s)\right)_{i, j, s=1,2} \in \mathbb{R}_{++}^{8}$. Therefore, if $N \subset \mathbb{R}_{++}^{8}$ is a (sufficiently small) neighborhood of $\bar{\nu}$, agent 1 is better off with financial market, but agent 2 is better off without financial market.

\section{The Dynamic Model}

In the previous section, we studied the effect of different markets on the allocation of consumption goods in the one-period world economy. In this static model, the initial endowment was completely consumed in the respective state at the end of the period. In what follows, we extend the analysis to 
a world economy with production and an infinite planning-horizon. If consumption goods are perishable and consumers have access to a production technology, consumers with a planning-horizon of more than one period have an incentive to save, because their investments generate consumption goods in the subsequent period. We need to specify how the investment decisions are made in each period. In our model, country $i$ owns the capital stock of good $i$ and specializes in the production of good $i$. Thus country $i$ 's total production determines the aggregate consumption of good $i$ in any period. Its savings determine future consumption. Therefore, country $i$ has an incentive to exercise market power in any market for good $i$. The goal of this section is to analyze the effect of different markets in this infinite-horizon optimal growth model with strategic interaction of both countries. The results in the static cases are used in the further analysis.

Due to the fact that each country is a monopolist for the good it supplies, we postulate the following structure in the process of decision making. In each country decisions are made at two distinct levels. On the one hand, there is a central planner in each country who chooses the amount of investment in each period and each state of the world. His objective is to maximize the discounted sum of utilities of the representative agent in his country over an infinite horizon. The planners' decisions, therefore, determine aggregate consumption of the respective goods in all states of the world and all periods. The savings are used as input for production, yielding consumption goods in the subsequent period. On the other hand, there are competitive (financial or spot) markets in each period which determine the allocation of the aggregate consumption between the two countries at the end of that period. The consumption good is perishable and all trades have to be fulfilled within one period; we exclude contracts with maturity after the recent period. We restrict our analysis to two extreme cases, which have also been studied in the one-period model: the sole presence of spot markets and the existence of a complete financial market.

When trading in the financial and spot markets, the representative agent in each country takes as given the aggregate consumption decision of the planner. As in the one-period case, this agent maximizes expected utility when trading on the financial market and state-dependent utility when trading on the spot market. In this process an indirect utility function for the representative agent in each country is derived. The indirect utility depends on prices and the initial endowment (the state-dependent aggregate consumption choices of the planner) of the representative agent as well as on 
the available markets (and asset structure). In each single period, prices are determined by the process of market clearing and therefore depend on the decisions of both planners. Thus, the allocation of the two goods at the end of each period is determined in a non-strategic fashion by the representative agents in the two countries, given the choice of the planner. Each planner, however, makes a strategic decision that takes account of both the trading decisions of the representative agent and the equilibrium price mechanism on financial and spot markets, given the other planner's policy.

Let us give a formal description of the above model. Denote by $k_{i t}$ the capital stock of country $i$ at beginning of period $t$. The initial capital stock is $k_{i 0}>0$. If $x_{i t}(s)$ is the state-dependent aggregate consumption of good $i$ in period $t$, then,

$$
k_{i(t+1)}=f_{i}\left(s, k_{i t}\right)-x_{i t}(s)
$$

is the investment choice, i.e. the capital stock at the beginning of period $t+1$ of country $i$ if state $s$ is revealed at time $t$. We assume that the exogenous production shock is an independent and identically distributed sequence of random variables.

The policy decision of the planner in country $i$ is a sequence of statedependent aggregate consumptions $\left\{x_{i t} \in \mathbb{R}_{+}^{2} \mid t \geq 0\right\}$. The indirect utility of the representative agent in period $t$ is given by the one-period utility introduced in Section 2. Let us focus on the financial market case, see Section 2.2 for the associated one-period model. The planner in each country is assumed to know the process of price-formation and thus perceives the associated equilibrium consumptions $c_{i j t}^{F}(s)$ as a function of the state-dependent aggregate consumptions $\left(x_{1 t}, x_{2 t}\right) \in \mathbb{R}_{+}^{4}$. The planner in country $i$ therefore knows the effect of his decision on the indirect utility of the representative agent in period $t$ which is given by,

$$
\nu_{i}^{F}\left(x_{i t}, x_{j t}\right)=\sum_{s=1}^{2} \pi_{s} u_{i}\left(s, c_{i 1 t}^{F}(s), c_{i 2 t}^{F}(s)\right),
$$

where $c_{i j t}^{F}(s)$ is given by the outcome in the respective one-period case, see (10). Under our specifications, $c_{i j t}^{F}(s)$ is uniquely determined by the preferences and the aggregate consumptions $\left(x_{1 t}, x_{2 t}\right) \in \mathbb{R}_{+}^{4}$.

The planners are strategic, taking account of the effect of their decision on the market-clearing prices through the effect of the representative agent's expected utility. However, the appearance of $x_{j t}$ in the indirect utility function 
of the representative agent of country $i$ represents a consumption externality because $x_{j t}$ is the state-dependent aggregate consumption decision of the planner in country $j$.

In order to the write down the intertemporal maximization problem of the planner we introduce some notation. Let $(\Omega, \mathcal{F}, \mathbb{P})=\left(S^{\infty}, \mathcal{B}(S)^{\infty}, \pi^{\infty}\right)$, $S=\{1,2\}$ denote the sample-path space of the exogenous shock. $\omega(t) \in S$ is the production shock at time $t$. As above, we refer to these shocks as $s \in S$ whenever atemporal aspects of the model are considered; no confusion should arise in what follows. For each $t, \mathcal{F}_{t} \subset \mathcal{F}$ is the $\sigma$-algebra containing the information on the shock up to (and including) time $t$. The planner chooses the state-dependent investment in any period $t$, given the information up to time $t$. Formally, a policy is a family of functions $\left\{x_{i t}: \Omega \rightarrow \mathbb{R}_{+} \mid t \geq\right.$ $0\}$, which is measurable with respect to $\mathcal{F}_{t}$, i.e., $x_{i t}$ depends only on the realization of shocks up to time $t$.

Planner $i$ chooses a policy that maximizes the discounted sum of indirect utilities, given the the other country's policy. Denoting the discount factor in country $i$ by $\delta_{i}$, the planner solves the problem,

$$
\begin{aligned}
\max _{\left\{x_{i t}: \Omega \rightarrow \mathbb{R} \mid t \geq 0\right\}} & \mathbb{E} \sum_{t=0}^{\infty} \delta_{i}^{t} \nu_{i}^{F}\left(x_{i t}, x_{j t}\right) \\
\text { s.t. } & k_{i(t+1)}=f_{i}\left(\omega(t), k_{i t}\right)-x_{i t}, \\
& 0 \leq x_{i t} \leq f_{i}\left(\omega(t), k_{i t}\right), \quad \text { for all } \omega \in \Omega, t \geq 0,
\end{aligned}
$$

given initial condition $k_{i 0}\left(\omega_{-1}\right) \equiv k_{i 0}>0$. For each $t \geq 0, k_{i t}$ is a random variable which is measurable with respect to $\mathcal{F}_{t-1}$.

The utility derived by the agent in country $i$ depends on the choices of the planners in both countries. This creates the possibility of strategic interaction. The strategic interaction comes through the process of equilibrium price formation on both financial and spot markets. In this form, the model is a differential game between the two planners.

In order to solve this game in stationary strategies and to obtain a closedform solution, we assume that the stochastic production function of country $i$ is given by

$$
f_{i}\left(s, k_{i}\right)=k_{i}^{\alpha_{i}(s)}, \quad 0<\alpha_{i}(s)<1 .
$$

A stationary strategy is a policy that depends only on the beginning-ofperiod capital stocks and on the possible realization of the state of the world in the recent period. 
Definition 3.1 A pair of functions $x_{1}, x_{2}: S \times \mathbb{R}_{+}^{2} \rightarrow \mathbb{R}_{+}$is a Cournot-Nash equilibrium in stationary strategies, if $x_{i}$ is an optimal policy for planner $i$ given the $j$ th planners policy $x_{j}$, for $i, j=1,2$ and $j \neq i$.

We determine policies which constitute a Cournot-Nash equilibrium in stationary strategies using stochastic dynamic programming and deriving a subgame-perfect Nash equilibrium. Let us discuss both spot and financial market case in turn.

\subsection{Spot Market Case}

In the spot market case, the indirect utility of the representative consumer in country $i$ in state $s$, which is perceived by the planner in country $i$ as a function of the aggregate consumption of both countries, is,

$$
\nu_{i}^{S}\left(s, x_{i}, x_{j}\right)=\eta_{i j}^{S}(s)+\nu_{i i}(s) \log x_{i}(s)+\nu_{i j}(s) \log x_{j}(s),
$$

$j \neq i$, where

$$
\begin{gathered}
\eta_{i j}^{S}(s)=\nu_{i i}(s) \log \frac{\nu_{i i}(s)}{\nu_{i i}(s)+\nu_{i j}(s)}+\nu_{i j}(s) \log \frac{\nu_{i j}(s)}{\nu_{i i}(s)+\nu_{i j}(s)} \\
+(-1)^{i} \nu_{i j}(s) \log \frac{\nu_{12}(s)\left(\nu_{21}(s)+\nu_{22}(s)\right)}{\nu_{21}(s)\left(\nu_{11}(s)+\nu_{12}(s)\right)}
\end{gathered}
$$

see the corresponding one-period case, Section 2.3.

The objective of the planner in country $i$ is to adopt a policy that maximizes the discounted sum of utilities of the representative agent, given the policy of the other country's planner. In each period, the planner makes his investment decision after the state of the world is known. However, the uncertainty in production implies that the endowment at the end of the next period is unknown at the time of decision making. The maximization problem of the planner is,

$$
\begin{aligned}
& \max _{\left(x_{i t}: \Omega \rightarrow \mathbb{R}\right)_{t \geq 0}} \mathbb{E} \sum_{t=0}^{\infty} \delta_{i}^{t} \nu_{i}^{S}\left(\omega(t), x_{i t}, x_{j t}\right) \\
& \text { s.t. } k_{i(t+1)}=k_{i t}^{\alpha_{i}(\omega(t))}-x_{i t}, \\
& \quad 0 \leq x_{i t} \leq k_{i t}^{\alpha_{i}(\omega(t))}, \quad \text { for all } \omega \in \Omega, t \geq 0 .
\end{aligned}
$$


We show next that a policy of choosing a state-dependent fraction of own output as aggregate consumption in every period is a Cournot-Nash equilibrium in stationary strategies. That is, in equilibrium, planner $i$ chooses $x_{i}\left(s, k_{1 t}, k_{2 t}\right)$ to be a linear function of $f_{i}\left(s, k_{i t}\right)$ such that, in particular, $x_{i}\left(s, k_{1 t}, k_{2 t}\right)$ is independent of the foreign countries capital stock $k_{j t}$. Adapting the technique of Fischer and Mirman [6] to compute a Cournot-Nash equilibrium, we proceed as follows. First, assume that a stationary value function $V_{i}\left(s, k_{i}\right)$ exists. Then the principle of optimality implies,

$$
\begin{aligned}
V_{i}\left(s, k_{i}\right)=\max _{0 \leq x_{i} \leq k_{i}^{\alpha_{i}(s)}}\left\{\eta_{i j}^{S}(s)\right. & +\nu_{i i}(s) \log x_{i}+\nu_{i j}(s) \log x_{j} \\
& \left.+\delta_{i} \sum_{\tilde{s}=1}^{2} \pi_{\tilde{s}} V_{i}\left(\tilde{s}, k_{i}^{\alpha_{i}(s)}-x_{i}\right)\right\} .
\end{aligned}
$$

By extrapolating from the corresponding finite-horizon version of the model, we assume a log-linear functional form,

$$
V_{i}\left(s, k_{i}, k_{j}\right)=A_{i}(s) \log k_{i}+D_{i}(s),
$$

where $A_{i}(s)$, and $D_{i}(s)$ depend only on the parameters of the model.

We demonstrate next that the value function (16) actually solves the functional equation (15). Assuming (16), the first-order condition yields,

$$
x_{i}(s)=\frac{\nu_{i i}(s)}{\nu_{i i}(s)+\delta_{i} \mathbb{E}\left(\alpha_{i} A_{i}\right)} k_{i}^{\alpha_{i}(s)},
$$

which shows that, in equilibrium, planner $i$ chooses $x_{i}\left(s, k_{1 t}, k_{2 t}\right)$ to be a linear function of its countries state-dependent aggregate output.

Now, one can check that this stationary policy actually solves the Bellman equation (15), where the parameter $A_{i}$ still has to be determined. Inserting the expression for the policy $x_{i}\left(s, k_{1}, k_{2}\right)$ into the optimality condition and then equating coefficients of $\log \left(k_{i}\right)$, one finds,

$$
A_{i}(s)=\nu_{i i}(s)+\delta_{i} \mathbb{E}\left(\alpha_{i} A_{i}\right),
$$

and some expression for $D_{i}(s)$, which is not needed for the further analysis.

We finally derive a closed-form solution for the optimal policy,

$$
x_{i}\left(s, k_{1}, k_{2}\right)=\gamma_{i}^{S}(s) k_{i}^{\alpha_{i}(s)}
$$


where the state-dependent optimal consumption-output ratio $\gamma_{i}^{S}(s)$ is given by,

$$
\gamma_{i}^{S}(s)=\left(1+\frac{\delta_{i}}{\nu_{i i}(s)} \frac{\mathbb{E}\left(\nu_{i i} \alpha_{i}\right)}{1-\delta_{i} \mathbb{E} \alpha_{i}}\right)^{-1} .
$$

It important to note that the optimal policy of the planner in country $i$ is independent of the policy of the planner in country $j$. This leads to the following result.

Proposition 3.2 The choice of investing a state-dependent proportion of capital stock, $1-\gamma_{i}^{S}(s)$, in every period by the planner in the country $i$, where $\gamma_{i}^{S}(s)$ is given by (18), is the unique subgame-perfect Cournot-Nash equilibrium in stationary strategies for the dynamic spot market case.

\subsection{Financial Market Case}

In the financial market case, the indirect utility of the representative agent in country $i$ perceived by the planner is, cf. Section 2.2,

$$
\nu_{i}^{F}\left(x_{i}, x_{j}\right)=\eta_{i j}^{F}+\sum_{s=1}^{2} \pi_{s}\left(\nu_{i i}(s) \log x_{i}(s)+\nu_{i j}(s) \log x_{j}(s)\right),
$$

where

$$
\eta_{i j}^{F}=\sum_{s=1}^{2} \pi_{s}\left[\nu_{i i}(s) \log \left(a_{i i}(s) \frac{d_{i 1}+d_{i 2}}{d_{i s}}\right)+\nu_{i j}(s) \log \left(a_{i j}(s) \frac{d_{i 1}+d_{i 2}}{d_{j s}}\right)\right] .
$$

We proceed analogously to the spot market case, Section 3.1, to solve the stochastic dynamic optimization problem (13). It is straightforward to show that the optimal consumption policy is,

$$
x_{i}(s)=\gamma_{i}^{F}(s) k_{i}^{\alpha_{i}(s)},
$$

where

$$
\gamma_{i}^{F}(s)=\left(1+\frac{\delta_{i}}{\nu_{i i}(s)} \frac{\mathbb{E}\left(\nu_{i i} \alpha_{i}\right)}{1-\delta_{i} \mathbb{E} \alpha_{i}}\right)^{-1}
$$

As in the dynamic spot market case, we find that the optimal policy of the planner in country $i$ is independent of the policy of the planner in country $j$. We therefore get the analogous result to Proposition 3.2: Investing a state-dependent proportion of capital stock, $1-\gamma_{i}^{F}(s)$, in every period in country $i$ is the unique subgame-perfect Cournot-Nash equilibrium in stationary strategies for the dynamic financial market case. 


\subsection{Cooperative Case}

To have a benchmark for the two dynamic cases analyzed in the previous sections, we derive the world equilibrium in the dynamic case next. In this equilibrium neither financial nor spot markets open. The integrated world equilibrium is the optimal cooperative equilibrium maximizing the weighted sum of the discounted expected utilities of the agents in the two countries. The objective of the single benevolent planner in the world is to choose feasible state-dependent aggregate consumptions in the two countries as well as an allocation of consumption goods for the representative agents in each country, for every period $t$, to maximize the weighted sum of the discounted expected utilities in both countries. This and the next section also extend the Brock and Mirman [3] analysis to a particular two-sector economy.

For any given aggregate consumption decision, the allocation of the consumption goods, in each period, solves the corresponding one-period maximization problem. Using (11), we find the indirect utility function,

$$
\nu^{C}\left(s, x_{1}, x_{2}\right)=\eta+\sum_{i=1}^{2}\left(\mu_{1} \nu_{1 i}(s)+\mu_{2} \nu_{2 i}(s)\right) \log x_{i}(s),
$$

where $\eta=\sum_{i, j, s=1}^{2} \frac{\mu_{i} \nu_{i j}(s)}{\mu_{1} \nu_{1 j}(s)+\mu_{2} \nu_{2 j}(s)}$.

Since the planner first observes the state $s$ and then decides on aggregate consumption and allocation, the optimization problem is as follows. For simplicity, we assume that $\delta_{1}=\delta_{2}=\delta$.

$$
\begin{aligned}
\max _{\left(x_{1 t}, x_{2 t}: \Omega \rightarrow \mathbb{R}\right)} & \mathbb{E} \sum_{t=0}^{\infty} \delta^{t} \nu^{C}\left(\omega(t), x_{1 t}, x_{2 t}\right) \\
\text { s.t. } & 0 \leq x_{i t} \leq k_{i t}^{\alpha_{i}(\omega(t))}, \\
& k_{i(t+1)}=k_{i t}^{\alpha_{i}(\omega(t))}-x_{i t}, \quad i=1,2, \text { for all } \omega \in \Omega, t \geq 0 .
\end{aligned}
$$

We proceed analogously to the spot market case. However, in the present case, the value function depends on the capital stock in both countries. We make the guess,

$$
V\left(s, k_{i}, k_{j}\right)=A(s) \log k_{i}+B(s) \log k_{j}+D(s) .
$$

It is then straightforward to find the optimal consumption policy,

$$
x_{i}(s)=\gamma_{i}^{C}(s) k_{i}^{\alpha_{i}(s)},
$$


where the consumption-output ratio in the efficient integrated world equilibrium for good $i=1,2$ is given by,

$$
\gamma_{i}^{C}(s)=\left(1+\frac{\delta}{\mu \nu_{1 i}(s)+(1-\mu) \nu_{2 i}(s)} \frac{\mathbb{E}\left(\left[\mu \nu_{1 i}+(1-\mu) \nu_{2 i}\right] \alpha_{i}\right)}{1-\delta \mathbb{E} \alpha_{i}}\right)^{-1}
$$

\subsection{Dynamic Efficiency of Allocations}

Dynamic efficiency of allocations requires that the share of the total stock of capital extracted in every period is identical to the benchmark consumption rate in the cooperative world-equilibrium. It is clear from our analysis that, given aggregate consumptions, the allocation of consumption goods in any period is identical to that derived in the one-period case. The Cournot-Nash equilibrium in stationary strategies, derived above for both non-cooperative cases, are compared to the cooperative benchmark-case. We assume that time preferences in both countries are equal, i.e. $\delta_{1}=\delta_{2}=\delta$.

The optimal aggregate consumption in each period, which is determined strategically by the planners, defines a stochastic law of motion for each countries' capital stock. For instance, in the financial market case, Section 3.2, the evolution of the capital stock of country $i$ is described by the stochastic process,

$$
k_{i(t+1)}=\left(1-\gamma_{i}^{F}(\omega(t))\right) k_{i t}^{\alpha_{i}(\omega(t))},
$$

where $\omega(t)$ is the realization of the state in period $t$, and $1-\gamma_{i}^{F}(s)$ is the optimal state-dependent investment-output ratio in state $s$, or saving rate, for short. We refer to $\gamma_{i}^{;}$, the optimal consumption-output ratio in the respective case, as the consumption rate in the following.

Let us compare first the consumption rates in the non-cooperative cases. Inspecting equations (18) and (20), one finds,

Lemma 3.3 The consumption rate (and, thus, the saving rate) is identical in the spot market case and financial market case, i.e. $\gamma_{i}^{S}(s)=\gamma_{i}^{F}(s)$ for $s=1,2$ and $i=1,2$.

This result says that the planners' decision is independent of the particular markets that are available to consumers. The amount of the domestic good extracted in every period by each countries planner is the same in both the spot and the financial market case. In particular, the amount of resources extracted every period does not depend on the availability of a financial market in which consumers can insure their wealth. 
The key to understanding why the planners choice is the same regardless of consumers particular trading possibilities is the maximization problem solved by each planner. Inspection of the indirect utility function that are perceived by the planner in each country yields the following observation. Maximizing the sum of discounted expected utilities in the financial market case, in which the indirect utility function is given by $\nu_{i}^{F}\left(x_{i}, x_{j}\right)$, yields the same (optimal) choice as maximizing with respect to the indirect utility function $\sum_{s} \nu_{i}^{S}\left(s, x_{i}, x_{j}\right)$ derived from the spot market case. Since the expected utility $\sum_{s} \nu_{i}^{S}\left(s, x_{i}, x_{j}\right)$ is maximal if and only if the state-dependent utility $\nu_{i}^{S}\left(s, x_{i}, x_{j}\right)$ is maximal for both states, the optimal choice is identical in the spot market and the financial market case. Note that in both the spot market case and the complete financial market case, the optimal consumption of each representative agent depends only on the aggregate consumption of his own good. However, the prices depend on the aggregate consumption of both goods. This is true in each state of the world. The planners are assumed to take account of the price formation process. This creates a consumption externality for each planner. However the properties of the utility and production functions in our model ensure that the consumption externality plays no role in the optimal choice, i.e., the optimal policies are in dominant strategies. It turns out that the optimal aggregate consumption choice of the planner in each country is a constant multiple of the stock of the good in that country. Although in the two market cases these multiples are different, due to our assumptions on the utility and production functions, they play no role in the dynamic (aggregate consumption) decisions. Therefore, we may conclude that in our model trading possibilities only effect the allocation of consumption goods within each period, but do not change the saving rate.

In the cooperative case the optimal consumptions also depend on the aggregate consumptions of both countries, as in the market cases. However, the dynamic decisions are different in the cooperative case since their the single central planner takes account of the consumption externality. Thus, these consumption externalities are 'internalized' by the single central planner. This internalizing of the consumption externalities yields efficient solutions, which can not be duplicated in the market cases unless the preferences are the independent of the state of the world or are essentially the same, but not necessarily identical, across consumers.

In order to illustrate these differences, we compare next the consumption rates in the non-cooperative cases and the cooperative case. Figure 1 illustrates the stochastic law of motion in the financial market case and the 

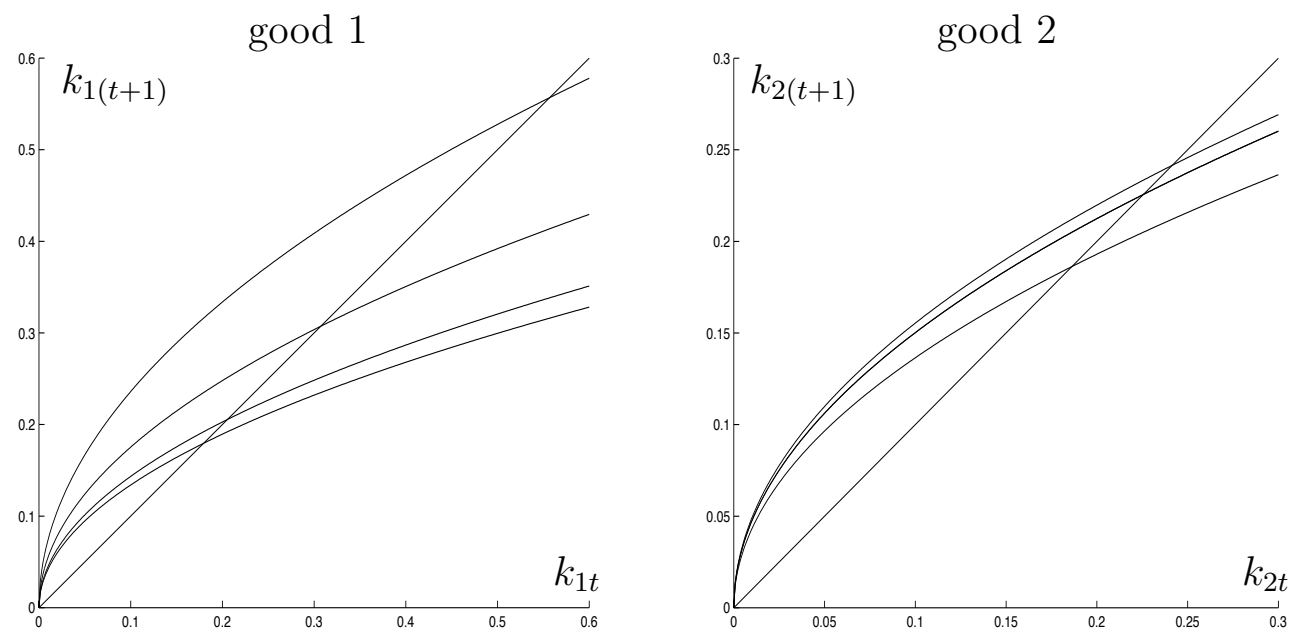

Figure 1: Illustration of the stochastic laws of motion for both goods in the financial market case and the cooperative case.

cooperative case. Parameters are fixed as follows: $\mu=.5, \delta=.95, \pi_{1}=.25$, $\alpha_{i}(s) \equiv .5, \nu_{11}(1)=.1, \nu_{11}(2)=.4, \nu_{12}(1)=.9, \nu_{12}(2)=.6$, and $\nu_{2 i}(s) \equiv .5$. In particular, the preferences of the representative agent in country 2 do not depend on the state. For these parameters, the laws of motion corresponding to the consumption rates have the following order (from top to bottom): $\gamma_{1}^{F}(1), \gamma_{1}^{C}(1), \gamma_{1}^{C}(2), \gamma_{1}^{F}(2)$ (left-hand side) and $\gamma_{2}^{C}(2), \gamma_{2}^{F}(1)=\gamma_{2}^{F}(2), \gamma_{2}^{C}(1)$ (right-hand side). Therefore, one finds that $\gamma_{1}^{F}(1)<\gamma_{1}^{C}(1)<\gamma_{1}^{C}(2)<\gamma_{1}^{F}(2)$, and $\gamma_{2}^{C}(2)<\gamma_{2}^{F}(2)=\gamma_{2}^{F}(1)<\gamma_{2}^{C}(1)$. Since the exponent $\alpha_{i}(s)$ is identical in all cases, one finds that for any fixed capital stock in period $t$, the capital stock in the subsequent period $t+1$ is the larger, the larger the saving rate (i.e. the smaller the consumption rate).

In the example, the most extreme saving rates for good 1 occurs in the financial market case. In this case, the saving rate for good 1 is largest in state 1 and smallest in state 2 . The saving rate in the cooperative case lies between these rates, but are larger in state 1 than in state 2 . In the longrun, one therefore observes a larger fluctuation of the capital stock of good 1 in the financial market case than in the cooperative case. The saving rate for good 2 shows a different pattern. Here, the saving rates in the financial market case are independent of the state. The saving rate in the cooperative case, however, varies with the state. It is smallest in state 1 and largest in state 2. For good 2, one therefore observes fluctuations of the capital stock 
only in the cooperative case, while in the financial market case the capital stock converges to a nonstochastic steady state in the long-run.

The over- resp. underconsumption in both goods relative to the cooperative case has the following causes. On the one hand, consumers in country 2 have state-independent preferences. The optimal choice of the planner is therefore to extract a fixed, i.e. state-independent, fraction of the total capital stock in each period. Consumers in country 1, on the other hand, have preferences that vary with the state. Since the planner in country 2 makes a different choice than a single world-planner, who would extract a state-dependent share of good 2, the planner in country 1 compensates for the relative over/undersupply of good 2 by extracting a smaller/larger share of its good in the respective state. In the cooperative case, the saving rates for good 1 and good 2 have a different order with respect to the state: $\gamma_{1}^{C}(1)<\gamma_{1}^{C}(2)$, and $\gamma_{2}^{C}(1)>\gamma_{2}^{C}(2)$.

If we change parameters such that $\nu_{11}(1)=.3$, and $\nu_{21}(1)=.9$, ceteris paribus, we find that $\gamma_{1}^{F}(1)<\gamma_{1}^{C}(2)<\gamma_{1}^{F}(2)<\gamma_{1}^{C}(1)$. The relation of consumption rates for good 2 are unchanged. For these parameters, the highest capital stocks of good 1 are observed in the financial market case, while the lowest ones occur in the cooperative case. We discuss in more detail the relation of the empirical distribution of the capital stocks in both non-cooperative and cooperative case at the end of this section.

Some comments on the relation of the saving rates and the correlation of preferences and production shocks are in order. Inspecting the defining equations for the consumption rates, it is clear that there is an unambiguous relation. If the shock $\alpha_{i}$ to the production of good $i$ and the preference of country $i$ for the domestic good are positively (negatively) correlated, then all consumption rates for good $i$ are lower (higher) than in the uncorrelated case. Moreover, in the cooperative case, the consumption rate is lower (higher) than in the uncorrelated case, if the preference of country $j$ for the foreign good is positively (negatively) correlated with the production shock $\alpha_{i}$.

The numerical example illustrates that the saving rate in the non-cooperative cases is, in general, different to that in the cooperative case. In such a situation, the allocation of goods cannot be dynamically efficient. We characterize next the situation in which the saving rate in the stationary Cournot-Nash equilibrium in both non-cooperative cases is identical to that in the cooperative world-equilibrium.

Proposition 3.4 The saving rate of good $i$ in the stationary Cournot-Nash 
equilibrium in both non-cooperative cases is equal to the saving rate in the cooperative case if and only if

$$
\frac{\nu_{i i}(1)}{\nu_{j i}(1)}=\frac{\nu_{i i}(2)}{\nu_{j i}(2)}
$$

where $i, j=1,2$, and $j \neq i$.

Proof. It is a simple exercise to check that the consumption rates defined in (20) and (23) are identical, if and only if (24) holds.

This result ensures that the saving rate for good $i$ in the non-cooperative Cournot-Nash equilibrium in both the spot-market and the financial-market case is efficient, if and only if the ratio of the preference of the two countries for good $i$ is the same across states. However, it is important to point out that efficiency of the saving rate and efficiency of the spot-market allocation in each period is not related: either of the conditions (12) and (24) can be fulfilled while the other is not satisfied.

If preferences of consumers exhibit constant returns for each state of the world, i.e. $\nu_{i i}(s)+\nu_{i j}(s)=1$ for all $i, j, s=1,2$, and $j \neq i$, then $(24)$ is equivalent to the condition that preferences of both consumers are identical.

Suppose the preferences of the representative agents in both countries for good $i$ do not depend on the state. Then the saving rate of good $i$ is efficient, i.e. it is identical to the saving rate in the cooperative case, Moreover, in this case the saving rate is also independent of the state and the allocation of consumption goods is Pareto-optimal, cf. Lemma 2.1.

We have already noted that the occurrence of country $j$ 's decision in the indirect utility function used by planner $i$ to determine his optimal strategic decision represents a consumption externality. The process of strategic interaction internalizes this externality, if the ratio of the preference of the two countries for good $i$ is the same across states. In any other case, the world economy fails to internalize this externality in the Cournot-Nash equilibrium. This observation has also been made by Datta and Mirman [4, Prop. 3] for state-independent preferences.

Let us study in detail the case in which the ratio of the preference of the two countries for good $i$ differs across states, i.e. the case in which condition (24) does not hold.

Proposition 3.5 Suppose that

$$
\frac{\nu_{i i}(s)}{\nu_{j i}(s)}>\frac{\nu_{i i}(\tilde{s})}{\nu_{j i}(\tilde{s})}
$$


for some $s, \tilde{s}=1,2, s \neq \tilde{s}$. Then there is overconsumption (underconsumption) of good $i$ in the non-cooperative case in state $s$ (state $\tilde{s}$ ), i.e. $\gamma_{i}^{F}(s)>\gamma_{i}^{C}(s)\left(\gamma_{i}^{F}(\tilde{s})<\gamma_{i}^{C}(\tilde{s})\right)$ for any weight $\mu \in(0,1)$, if and only if (25) holds.

Proof. Again it is left to the reader as an easy exercise to check that the consumption rates defined in (20) and (23) have the claimed property, if and only if (25) holds.

This result shows that if preferences do not satisfy (24) then there is either overconsumption or underconsumption in the sense that aggregate consumption is lower/higher than in the cooperative case. Proposition 3.5 further ensures that it is not possible to have either over- or underconsumption in both states. If the saving rate is not efficient, then, in one state of nature, there is overconsumption whereas there is underconsumption in the other state. However, it may well be that the saving rate of one good is efficient, while it is inefficient for the other good.

We close the section with a discussion on the relation of the empirical distribution of the capital stocks in the two cases. In the non-cooperative cases, the consumption rate $\gamma_{i}^{S}(s)=\gamma_{i}^{F}(s)$ depends only on the preferences of country $i$ for the domestic good. However, the consumption rate $\gamma_{i}^{C}(s)$ in the efficient case depends on the preferences of both countries for good $i$. Therefore, there is no general relation between these consumption rates across 'market' cases as well as across goods and, thus, the time-series of the capital stocks are not related in a particular way. The only relation that holds can be drawn from Propositions 3.4 and 3.5: The consumption rates for each good $i$ satisfy $\gamma_{i}^{F}(s) \geq \gamma_{i}^{C}(s)$, and $\gamma_{i}^{F}(\tilde{s}) \leq \gamma_{i}^{C}(\tilde{s})$, for some $s, \tilde{s}=1,2$, $s \neq \tilde{s}$.

To further analyze the empirical distribution we need some notation. It is straightforward to check that, for each fixed state $s$, the map $k \mapsto h_{i}^{F}(s, k)=$ $\left(1-\gamma_{i}^{F}(s)\right) k^{\alpha_{i}(s)}$, which is derived from the stochastic law of motion, has a unique positive fixed point. Denote the fixed points for $s=1,2$ by $\underline{k}_{i}$ and $\bar{k}_{i}$, where $\underline{k}_{i} \leq \bar{k}_{i}$. Since $h_{i}^{F}(s, k)$ is concave and increasing, the interval $G_{i}^{F}=\left[\underline{k}_{i}, \bar{k}_{i}\right]$ is forward-invariant under the stochastic law of motion, i.e. $h_{i}^{F}\left(1, G_{i}^{F}\right) \cup h_{i}^{F}\left(2, G_{i}^{F}\right) \subset G_{i}^{F}$. The same arguments apply in the cooperative case, yielding a forward-invariant interval $G_{i}^{C}$. In the long-run, the time-series of the capital stock of good $i$ is in $G_{i}^{F}$ resp. $G_{i}^{C}$ (if the intervals are proper). It can further be shown, see Schenk-Hoppé and Schmalfuss [9], that the timeseries of each of the two capital stocks are identical in the long-run for all 
positive initial capital stocks. In particular the empirical distribution is welldefined, i.e., independent of the initial capital stock, see also Mirman [7]. The support of the empirical measure is a subset of the forward-invariant interval, defined above. The relation between the consumption rates, stated above, implies that $G_{i}^{F} \cap G_{i}^{C} \neq \emptyset$. Therefore, in the long run, capital stocks in one case cannot be systematically higher than in the other case. However, the lowest resp. highest capital stocks observed in the long run may be associated with one of the cases, non-cooperative or cooperative.

It might be of independent interest to note that the support of the empirical measure is either a compact interval (here, $G_{i}^{F}$ resp. $G_{i}^{C}$ ) or a Cantor set. The latter case is observed if $h_{i}^{F}\left(1, G_{i}^{F}\right) \cap h_{i}^{F}\left(2, G_{i}^{F}\right)=\emptyset$. This result can be derived completely analogous to the corresponding case in the theory of Iterated Function Systems, see e.g. Barnsley [2, Chap. 4.5].

\section{References}

[1] K. J. Arrow, Le rôle de valeurs boursières pour la rèpartition la meilleure des risques. Econométrie (1953), 41-48. Translated as: The role of securities in the optimal allocation of risk-bearing, Review of Economic Studies 31 (1964) 91-96.

[2] M. Barnsley, Fractals Everywhere, New York: Academic Press (1988).

[3] W. Brock and L. J. Mirman, Optimal economic growth and uncertainty: the discounted case, Journal of Economic Theory 4 (1972) 479-513.

[4] M. Datta and L. J. Mirman, Dynamic externalities and policy coordination, Review of International Economics 8 (2000) 44-59.

[5] G. Debreu, Theory of Value, New York: Wiley (1959).

[6] R. D. Fischer and L. J. Mirman, Strategic dynamic interaction, Journal of Economic Dynamics and Control 16 (1992) 267-287.

[7] L. J. Mirman, The steady state behavior of a class of one sector growth models with uncertain technology, Journal of Economic Theory 6 (1973) 219-242. 
[8] R. Radner, Equilibrium under uncertainty. Chapter 20 in Handbook of Mathematical Economics, ed. K. J. Arrow and M. D. Intriligator, Volume II, Amsterdam: North-Holland, 923-1006 (1982).

[9] K. R. Schenk-Hoppé and B. Schmalfuss, Random fixed points in a stochastic Solow growth model, Working Paper No. 65, Institute for Empirical Research in Economics, University of Zurich, November 2000, revised version. 